\title{
Enhanced Spectral Utilization of 3G WCDMA-Based FDD Mode in the Uplink Transmission
}

\author{
Joseph Isabona \\ Department of Basic Sciences, Benson Idahosa University \\ PMB. 1100, Benin City, Benin, Nigeria \\ Tel: 23-4-703-918-1006Ｅ-mail: josabone@yahoo.com
}

Moses Ekpenyong

Department of Computer Science, University of Uyo

PMB. 1017 520003, Uyo, Akwa Ibom State, Nigeria

Tel: 23-4-803-793-3961_E-mail: ekpenyong_moses@yahoo.com, mosesekpenyong@gmail.com

Samuel Azi

Department of Physics, University of Benin

PMB. 1100, Benin City, Benin, Nigeria

Tel: 23-4-803-239-5000Ｅ-mail: ugochukwuazi@gmail.com

\begin{abstract}
The economic value on network capacity has made it a major determinant in the design of any generation of mobile technologies. For any multi-user cellular system, the measure of its economic usefulness is the peak load that can be supported within a given quality and service (QoS) availability. In this paper, we improve upon the existing Wideband Code Division Multiple Access (WCDMA) user capacity expressions in single and multi cell environments for the uplink, by integrating new parameters that affect the system. Specifically, we study and report the effect of multi-user detection and adaptive antenna gain on WCDMA users' capacity in the presence of loading, voice activity, sectorization, power control factor and bandwidth efficiency.
\end{abstract}

Keywords: Multiuser detection, Adaptive antenna gain, Network capacity, WCDMA, QoS

\section{Introduction}

Recently, the telecommunication industries have witnessed a phenomenal growth in the development and deployment of wireless services. This is evident from the proliferation of cellular data services and emerging wireless multimedia applications. This proliferation has opened up new research avenues and calls for the re-examination of some fundamental issues in wireless cellular networks. One major fundamental issue to address is the provision of higher capacity cellular systems with guaranteed QoS to meet its daily service demands for both commercial and residential subscribers. Although the second generation $(2 \mathrm{G})$ wireless systems such as the Global System for Mobile Communications (GSM) and IS-95, are successful in many countries (Jin and Yu-Dong, 2003), they still do not meet the requirement of high speed data and user capacity in densely populated areas.

Higher system capacity, better QoS and flexible accommodation of diverse wideband services such as video and multimedia services with different transmission rates are required in third generation (3G) wireless communication systems (Kim and Adachi, 2001). Code Division Multiple Access (CDMA) has been chosen as the radio interface technology for 3G systems (IEEE Comm. Magazine, 1999), unlike Frequency Division Multiple Access (FDMA) and Time Division Multiple Access (TDMA), which primarily are either bandwidth or dimension limited in capacity (Gilhousen, Jacobs, Padovani, Viterbi, Weaver and Wheatley, 1991). Some benefits offered by this technology include high data rates, universal and low-cost terminals, global standard, seamless roaming and interoperability. The technology is used in Ultra High Frequency (UHF) cellular phone systems within $800 \mathrm{MHz}-1.9 \mathrm{GHz}$ frequency range.

Contrary to FDMA and TDMA systems, the main limiting factor for capacity expansion in WCDMA system is the interference (De Sousa, De Lima, Rodrigues, Cavalcanti and Braga, 2003). Each user acts as an interference source to other users in the system. As such, a reduction in interference will directly increase the capacity. 
Emerging technologies such as directional adaptive antenna, rake receivers, and multi-user detection, could lead to a significant reduction in the interference and result in many fold capacity increase (Tse and Hanly, 1999, Ammer, 2004, Amitava and Saha, 2004). Capacity estimation is therefore an important element in the design of CDMA systems and in the performance evaluation of emerging technologies.

Several attempts at formulating, modeling and evaluating capacity equations for WCDMA cellular networks have been made. In CDMA performance evaluations, the user capacity is used to measure the CDMA system, with continuously active users (Kim and Honig, 2000). By user capacity, we refer to the number of users a CDMA system can support at a desired signal-to-noise ratio (SNR) satisfying certain conditions (Alma, 2008). In Gilhousen et. al (1991), the CDMA uplink user capacity is estimated considering voice traffic only, where strength-based power control (Xia, Shroff and Chong, 2001, Isabona and Ekpenyong, 2009, El-Osery and Abdullah, 2000) is assumed and the total other-cell interference, $I_{\text {other }}$, is modeled as a Gaussian noise. $I_{\text {other }}$ increases with the number of active users per cell, K, which decreases the SNR. The effect of rake receiver and antenna diversity on reverse link user capacity is presented in Kim and Adachi (2001), where the received signals at different antenna groups are assumed to be independent of each other. Also, modification and throughput optimization of WCDMA capacity equations are studied in Alma (2008), Ekpenyong, Isabona and Akpaeti (2009). However despite its advantages, there still remain challenges (complexities associated with detection and degradation in performance) in the design of WCDMA receivers, arising from Multiple Access Interference (MAI), which limits the system's capacity.

In this paper, we modify the existing WCDMA capacity expressions for the uplink transmission, integrating more parameters, which affect its operations. A closed-form equation relating multi-antenna gain, multi-user detection, power factor, sectorization factor, voice activity factor, bandwidth efficiency factor, target SNR and CDMA processing gain is presented and studied through extensive computer simulation to estimate the uplink user capacity.

\section{System Model for Uplink Capacity}

The capacity of a CDMA cell is determined by the balance between the required signal-to-noise-ratio (SNR) for each user, and the spread spectrum processing gain. The notation that determines the merit of a well-designed digital receiver is the dimensionless SNR given in equation (1):

$$
\frac{\varepsilon_{b}}{N_{0}} \equiv \frac{\text { Energy per bit }}{\text { Power spectral density noise }+ \text { Interference }}
$$

The "noise" part of the SNR in a spectrum system is actually the sum of the thermal noise and the other-user Interference. The energy-per-bit, $\varepsilon_{b}$ is related to the signal power $P_{r}$, and data rate, $R_{b}$ as given in equation (2):

$$
\varepsilon_{b}=\frac{P_{r}}{R_{b}}
$$

The noise-power spectral density, $N_{0}$, is the ratio of the total interference power, $I$ to the transmission bandwidth $W$, as expressed in equation (3):

$$
N_{0}=\frac{I}{W}
$$

Given the above relations, the bit-energy-to-noise density can be rewritten as:

$$
\varepsilon_{b} / N_{0}=\left(\frac{P_{r}}{F}\right) \cdot\left(\frac{W}{R_{b}}\right)
$$

where the ratio $\frac{W}{R_{b}}$ indicates the processing gain of the system.

Let us consider a single cell load with $K$ users that are in power control with the base station. Following the derivation in Garg and Johnson (2000), the total interference power for user $i$, is composed of the received signal power $P_{r j}$ from all users in the cell:

$$
I=\sum_{j=1, j \neq i}^{K} \frac{P_{r i}}{W}+N_{t h}
$$

where $N_{t h}$ is the thermal noise. If the power of signals of all active users have the same value, equation (5) simplifies to 


$$
I=\frac{(K-1) P_{r}}{W}+N_{t h}
$$

Substituting equation (6) into (4), the $\varepsilon_{b} / N_{0}$ on the uplink, allotted to an arbitrary user is

$$
\frac{\varepsilon_{b}}{N_{o}}=\frac{W}{R} \cdot \frac{P_{r}}{N_{t h} W+(K-1) P_{s}}
$$

Ignoring the thermal noise $N_{t h}$ and solving for $K$ in equation (7), yields the network capacity (number of users) in the system

$$
K=1+\frac{P G}{\varepsilon_{b} / N_{0}}
$$

where $_{P G}=W / R$.

Equation (8), which defines the number of simultaneous active users, is the basis for the WCDMA capacity. If we take into account the background thermal noise $N_{t h}$ over the full bandwidth $N_{0} W$ (see equation (7)), then the number of active users is reduced by the inverse of the signal-to-noise-ratio (SNR) in the total system bandwidth. Thus

$$
K=1+\frac{P G}{\varepsilon_{b} / N_{0}}-\frac{N_{t h} W}{P_{r}}
$$

\section{Impact of Different Parameters on the Capacity Model}

The analysis takes into account a number of capacity influencing factors such as loading factor, sectorization, voice activity factor, power factor and the effect of intra and inter interference. This paper does not explicitly address modulation techniques and their performance; rather, it assumes an efficient modulation and forward error correcting coding for given channels under established conditions, which the receiver achieves an acceptable level of performance, particularly in terms of the maximum number of users supported per cell.

\subsection{Loading}

Equation (9) is an effective model that describes the number of active users a single WCDMA cell can support, without any interference from neigbouring cells. To roughly include the effects from surrounding cells, we require the introduction of a loading factor $\eta$. This term indicates the increase in interference above the inverse of the factor $(1+\eta)$, known as the frequency reuse factor $F$. $F$ is related to the loading factor $\quad \eta$, by

$$
F=\frac{1}{1+\eta}
$$

For a single cell, the reuse factor is unity and the loading is zero. When multiple cells are considered, cell loading increases while $F$ decreases. If we include the loading factor $\eta$ from other cells, equation (7) becomes

$$
\frac{\varepsilon_{b}}{N_{0}}=P G \frac{P_{r}}{N_{t h} W+(K-1) P_{r}(1+\eta)}
$$

Again, we can solve for K:

with $W / R=P G$

$$
K=1+P G\left(\frac{1}{\varepsilon_{b} / N_{0}(1+\eta)}\right)-\frac{N_{t h} W}{P_{r}(1+\eta)}
$$

\subsection{Antenna sectorization}

Let $N_{s}$ represent the number of sectors in the cell-sector antenna. The use of sectorization and directional antennas could increase the capacity because it reduces interferences (Tse and Hanly, 1999). For instance, a cell site with three directional antennas, each having a perfect beam-width of $120^{\circ}$, has interference which is one-third of the interference in the case of an omni-directional antenna.

\subsection{Voice activity factor}

CDMA use speech coding, reducing the rate of the speech coder with voice activity detection along with variable rate transmission, could decrease the multiple access interference. By employing a variable rate vocoding, the 
system reduces the total interference power by the voice activity factor $v$. In Ammer (2004), the voice activity factor of human speech is approximately $42 \%$.

\subsection{Power factor}

Because the performance of WCDMA system is interference limited, we require accurate power control (Suyeb, Jasvir and Mian, 2006, Ernestina, 2005). An increasing power control error will reduce the system's capacity. The value of the power control error will depend on the system implementation and on the algorithm used in the control. This could be defined using a log-normal distribution with suitable power correction factor $\alpha_{p}$ (Karimm and Sarraf, 2005). So, we modify equation (11) to account for the effect of the voice activity factor, $v$, power factor, $\alpha_{p}$ and antenna sectorization, $N_{s}$, to have

$$
\frac{\varepsilon_{b}}{N_{0}}=\frac{N_{s} \alpha_{p}}{v} \frac{P G}{(K-1)(1+\eta)+\frac{N_{t h} W}{P_{r}}}
$$

We then extract a parametric formula for the capacity, thus

\subsection{Bandwidth efficiency factor}

$$
K=\frac{N_{s} \alpha_{p}}{v}\left[\frac{P G}{\varepsilon_{b} / N_{0}(1+\eta)}\right]-\frac{N_{t h} W}{P_{r}(1+\eta)}+1
$$

The bandwidth efficiency factor $\beta_{F}$ is introduced here because the individual users in a cell do not necessarily take the entire frequency band available to the system. Thus, if there are $K$ users inside the cell, which are homogeneously distributed over the frequency carriers of the cell, the number of users of the same frequency band is $K / \beta_{F}$ and the number of interferers from the cell is given by

$$
\frac{K}{\beta_{F}}-1
$$

This transforms equation (13) into

$$
\frac{\varepsilon_{b}}{N_{0}}=\frac{N_{s} \alpha_{p}}{v} \frac{P G}{\left(\frac{K}{\beta_{F}}-1\right)(1+\eta)+\frac{N_{t h} W}{P_{r}}}
$$

Taking into account the bandwidth efficiency factor, $\beta_{F}$ with other capacity enhancement parameters, we obtain an expression for $\mathrm{K}$ :

$$
K=\beta_{F}\left[1+\frac{P G}{\varepsilon_{b} / N_{0}}\left(\frac{1}{1+\eta}\right) \frac{\alpha_{p} N_{s}}{v}\right]-\frac{N_{t h} W}{P_{r}(1+\eta)}
$$

We are interested in equations (8), (9), (12), (13) and (17).

\section{The Impact of More Capacity Improvement Parameters on WCDMA Capacity Model}

The use of various interference elimination methods could increase system capacity. Some of the methods include use of directional antenna processing system, rake receivers, multi-user detection, etc.

\subsection{Adaptive antenna}

Interference due to other users can be reduced by replacing an omni-directional antenna with a directional antenna. For instance, a cell site with three directional antennas, each having a perfect beam-width of $120^{\circ}$ has interference which is one-third of the interference in an omni-directional antenna (Alma, 2008). Use of adaptive (multi) antenna system with the above assumptions will increase the WCDMA capacity, so the parameter $A_{d}$, which describes antenna gain of referent users to antenna gain of its jammers ratio could be input directly into equation (16). We now modify equation (17) as another suggested expression to show the impact of the usage of multi antenna system $\left(A_{d}\right)$ on the overall capacity, thus

$$
K=\frac{B_{r} A_{d} \alpha_{p} N_{s}}{V_{f}}\left[P G\left(\frac{1}{\varepsilon_{b} / N_{0}(1+\eta)}\right)\right]-\frac{N_{0} W}{\operatorname{Pr}(1+\eta)}+1
$$

$A_{d}$ could be easily related with the number of antenna elements $\mathrm{B}$, as 


$$
a_{b}[d B]=10 \log B
$$

where $a_{b}$ is the antenna gain expressed in $\mathrm{dB}$.

\subsection{Multi-user Detection (MUD)}

A major technological difficulty in CDMA systems is the 'near far' problem (i.e. strong signal swamping weaker signal) and Multiple Access Interference (MAI). MUD provides a means of reducing the effect of multiple interference and hence increases the system's capacity. The optimum multi-user detector for asynchronous multiple access Gaussian channels is obtained in Ammer (2004), where the 'near far' problem suffered by the convectional CDMA receiver, accounts for the presence of other interference in the channel. Thus

$$
\frac{\varepsilon_{b}}{N_{0}} \frac{P G}{(1-\beta) I_{\text {Intra }}+I_{\text {Inter }}+\frac{N_{t h} W}{P_{r}}}
$$

where $\beta$ denotes the capacity enhancement parameter of the multi-user detection technique. We assume here that the multi-user receiver accepts only those users (Intra-cell users) whose interference the MUD has cancelled. This implies that in a practical system, the capacity will be limited by the efficiency of the algorithm and the inter-cell interference. If Intra cell interference $I_{\text {Intra }}$ is taken to be $(K-1) P_{r}$, equation (19) yields

$$
\frac{\varepsilon_{b}}{N_{0}}=\frac{P G P_{r}}{(1-\beta)(K-1) P+I_{\text {Inter-cell }}+N_{\text {th }}}
$$

The Inter-cell interference is proportional to Intra-cell interference by the relation

$$
\text { Inter }=\eta \operatorname{Intra}=\left(\frac{1}{F}-1\right) \text { Intra }
$$

Substituting equation (21) into (20), we have

$$
\varepsilon_{b} / N_{0}=\frac{P G P_{r}}{(1-\beta)(K-1) P_{r}+\left(\frac{1}{F}-1\right)(K-1) P_{r}+N_{t h} W}
$$

since $I_{\text {Intra }}=(K-1) P_{r}$.

From equation (22), the uplink network capacity to meet required $\varepsilon_{b} / N_{0}$ becomes

\subsection{Capacity gain}

$$
K=\frac{\frac{\beta_{F} N_{S} \alpha_{P}}{V}\left[1+P G\left(\frac{1}{\varepsilon_{b} / N_{0}(1+\eta)}\right)\right]-\frac{\beta_{F} N_{t h} W}{P_{r}(1+\eta)}}{(1+\beta)+\left(\frac{1}{F}-1\right)}
$$

An analytical capacity gain estimation with multi-user detection is derived in this section. The total noise level spectral density $N_{0}$ at the base station generated by thermal noise $N_{t h}$ of all interferers from inside the cell $I_{\text {Intra }}$ plus noise generated by all interferers from outside the $I_{\text {Intra }}$ is

$$
\begin{aligned}
N_{0} & =N_{\text {th }}+I_{\text {Intra }}+I_{\text {Inter }} \\
& =(1+\eta) \text { Intra }+N_{0}
\end{aligned}
$$

The noise-rise is defined as the ratio of the total noise level spectral density to the thermal noise:

$$
\text { Noise rise }=\frac{N_{0}}{N_{t h}}
$$

The wideband interference after MUD can be written as

$$
N_{0(t o t a l, M U D)}=(1+\eta-\beta) I_{\text {Intra }}+N_{\text {th }}
$$

where we assume that $M U D$ is able to cancel part of the intra-cell interference $I_{\text {Intra. }}$. The cancelled part is denoted by

$$
\beta=\text { MUD efficiency }
$$

When evaluating the capacity gain, we assume that the mobile transmission power remains constant, i.e. the coverage of the cell is not affected. This requires that the interference power after multi-user detection, $N_{O_{(\text {total,MUD) }}}$ remains the same as without multi-user detection $N_{\text {o(total) }}$.

$$
N_{0(\text { total }, M U D)}=N_{0 \text { (total })}
$$

The capacity gain can be obtained using equations (23) and (25)

$$
(1+\eta-\beta) \bullet \text { Capcity gain } \bullet I_{\text {Intra }}+N_{\text {th }}=(1+\eta) I_{\text {Intra }}+N_{\text {th }}
$$


Solving equation (29) yields the capacity gain:

\subsection{Coverage gain}

$$
\text { Capacity gain }=\frac{1+\eta}{1+\eta-\beta}
$$

The coverage gain is evaluated with the assumption that the number of active users remains constant, i.e. the capacity of the cell is not affected. The coverage gain is defined as the decrease in the active user output power, which alternatively could be used to extend the cell range. The noise power $N_{\text {o(total,MUD) }}$ after MUD is derived as follows

$$
N_{0(\text { total }, M U D)}=(1+\eta-\beta) \frac{I_{\text {Intra }}}{\text { Coverage gain }}+N_{\text {th }}
$$

The coverage gain is equal to the reduction in the noise power after MUD:

$$
\begin{aligned}
& \text { Coverage gain }=\frac{N_{0(\text { total })}}{N_{0(\text { total }, M U D)}} \\
& \text { Coverage gain }=\frac{(1+\eta) \cdot I_{\text {Intra }}+N_{\text {th }}}{(1+\eta-\beta) \cdot \frac{I_{\text {Intra }}}{\text { Coverage gain }}+N_{\text {th }}} \\
& \text { Coverage gain }=\frac{(1+\eta) \cdot \frac{I_{\text {Intra }}}{N_{\text {th }}}+1}{(1+\eta+\beta) \cdot \frac{1}{\text { Coverage gain }} \cdot \frac{I_{\text {Intra }}}{N_{\text {th }}}+1}
\end{aligned}
$$

Merging equations (24) and (25) gives

$$
\frac{I_{\text {Intra }}}{N_{\text {th }}}=\frac{\text { Noise rise }-1}{1+\eta}
$$

Solving for Coverage gain using equations (32) and (33) gives

$$
\text { Coverage gain }=1+\frac{\beta(\text { Noise rise }-1)}{1+\eta}
$$

\section{System Performance and Discussion of Result}

Empirical data used for simulating the impact on WCDMA capacity, including its system design parameters are shown in Table 1. Some of these values have been used in Alma (2008), Isabona (2008), Isabona and Ekpenyong (2009), to obtain a fairly improved performance. The empirical data were gathered from real life simulation experiments using well established conditions.

Fig. 1 illustrates how number of active users varies with the signal-to-noise ratio (SNR), in the presence of other parameters. When we isolate other parameters and consider the impact of processing gain and the SNR on the system, the system supports only 11 users at $\varepsilon_{b} / N_{0}=9 \mathrm{~dB}$ for voice applications, without any performance effecting parameter. Due to the loading factor on desired users by neighbouring cells, the capacity of the system degrades to about 5 users. After the introduction of induced capacity enhancement parameters such as sectorization, voice activity factor, power control factor and adaptive antenna gain, the system performance improves, thus supporting 15, 17, 21 and 25 users, with all the enhancement parameters. Also, with power and bandwidth efficiency factors, the system supports 12 and 14 users respectively. The improvement in system performance with adaptive antenna leads other parameters (see Fig. 1.). The improvement in SNR is about $3 \mathrm{~dB}$ with two antenna elements, $6 \mathrm{~dB}$ with four elements, $8 \mathrm{~dB}$ with six elements and $9 \mathrm{~dB}$ with eight elements. Obviously, the implementation of multi-antenna system will reduce the average interference level from all users particularly, so the total channel capacity increases.

The expression in equation (17) could also be applied when comparing values of WCDMA capacity depending on SNR of the different antenna gain ratio. This is shown in Fig. 2. Therefore, for a desired SNR of 9 or $6 \mathrm{~dB}$ (for a BPSK or QPSK with coherent detection), the number of simultaneously active users for one service is 20 for $A_{d}=2.5$, for instance. 
Fig. 3 shows the users capacity enhancement with and without MUD. A comparison of the combined effects of various parameters with the capacity enhancement MUD technique $(\beta)$ in multi cell environments is shown in Fig. 4. This technique minimizes the multiple access interference and increases the performance up to 22 users per cell, considering same set of parameters in Fig. 3. To study the impact of different operations scenario, different multi-user detection factor is assumed to represent different multi-path propagation. The effect is shown in Fig. 4. We observe in Fig. 4 that, when MUD is increased, more users can access the same channel bandwidth simultaneously. This is represented using equation (23).

Assuming a MUD factor of $60 \%$ and typical macro cellular other-to-own cell interference ratio of 0.65 , we obtain a capacity gain of $65 \%$. The capacity gain as a function of efficiency is shown in Fig. 5. In a micro cellular environment, the cells tend to have a higher isolation, making other-to-own cell interference ratio smaller. With a cell interference ratio of 0.3 , and assuming a MUD of $70 \%$, a capacity gain of $80 \%$ is obtained in micro cellular environments.

The coverage gain as a function of the initial noise rise is shown in Fig. 6, assuming a MUD factor of $65 \%$ and other-to-own cell interference ratio of 0.65 . The coverage gain of the MUD receiver improves the coverage from $1.2 \mathrm{~dB}$ to $2 \mathrm{~dB}$. This is shown in Fig. 7. Here a link level improvement of $1 \mathrm{~dB}$ can be used to increase the cell area by $15 \%$.

Capacity is related to the spectral efficiency (SE) of a system, as well as the amount of traffic offered by each user. The spectral efficiency (Hosham, Salim and Hessein, 2008) is given by

$$
S E=\text { Network capacity } \times(\operatorname{Pr} \text { oces } \sin g \text { gain })^{-1}[[\text { b/s }) /(\mathrm{Hz})]
$$

From the results shown in Fig. 8, it is clearly observed that the various capacity enhancement parameters have potentially increased the spectral efficiency. We attribute this to the reduced interference, which allows the users to operate at higher rate.

\section{Conclusion and Outlook}

Enhanced spectral utilization allows the maximum number of users with adequate signal strength in a CDMA cell. This paper has modified the basic expression of WCDMA capacity with elements that impact significantly on it. A modified expression for WCDMA computation has been proposed. The use of multi antenna (adaptive) system and multi user detection as methods for increasing the system capacity are strongly recommended as promising solutions for future generation mobile communication systems (for both macro and micro cellular environments) to provide improved system performance and dependable quality of service to both residential and commercial subscribers (or end-to-end users).

So far, our CDMA system capacity modeling optimization approach considers the various parameters such as MUD, adaptive antenna, etc., which constitute the main features of the physical layer. We also assumed efficient coding and modulation. In reality, however, time varying channel conditions could cause the propagated signal to fluctuate at some degree. Though this can be resolved through efficient coding and modulation methods, is beyond the scope of this paper and is slated for a future paper.

\section{References}

Alma, S. R. (2008). The Modification of WCDM Capacity equation. $16^{\text {th }}$ Telecommunication Forum, Telfor. 266 -269 .

Amitava, M. and Saha, D. (2004). Approaches for Radio Resource Management in Mobile Wireless Networks: Current Status and Future Issues. Technical reports (University of New South Wales. School of Computer Science and Engineering); UNSW-CSE-0404.

Ammer, C. (2004) Multiuser Detection Techniques for UMTS. Bechtel Telecommunication Journal. 2(2): 1-2.

De Sousa, V. A., De Lima, C. H. M, Rodrigues E. B, Cavalcanti F. R. P and Braga A. R. (2003): Coverage and Capacity of WCDMA Systems with Beam and Steering Antenna. Vehicular Technology Conference, VTC 2003 Fall, 2:826-830.

Ekpenyong, M., Isabona J., and Akpaeti, A. (2009): Robust Modelling and Throughout Optimization in CDMA Networks. Journal of Applied Science and Engineering Technology. 3: 5-12.

El-Osery A, and Abdullah C. (2000). Distributed Power Control in CDMA Cellular Systems. IEEE Antennas and Propagation Magazine. 42(4): 152-159. 
Ernestina, C. (2005) Spread Spectrum Technique and Their Applications to Wireless Communications. IETE Journal of Research, 15(1): 5-18.

Garg V. K. and Johnson K. U. (2000). IS-95 CDMA and CDMA 2000. Upper saddle Rius. NJ Prentice Hall.

Gilhousen K S., Jacobs, I. M., Padovani, R. Viterbi, A. J., Weaver, L. A. and Wheatley, C. E. (1991): On the capacity of a cellular CDMA System. IEEE Transactions on Vehicular Technologies. 40(2): $303-312$.

Hosham S. S. and Hessein B. (2008). Performance Enhancement of GSM Cellular Phone Network using Dynamic Frequency Hopping, Engineering and Technology. 26(3): 365 - 375.

IEEE Communication Magazine (1999). Special Issue on Wideband Code Division Multiple Access WCDMA 36.

Isabona J. (2008). Transmit Power Control in Multimedia CDMA Cellular System. M.Sc. Thesis, University of Uyo, Nigeria.

Isabona, J. and Ekpenyong, M. (2009) Performance Evaluation of Coverage Capacity Interaction in CDMA Wireless Networks. Journal of the Nigerian Mathematical Society. 28: 153 - 167.

Jin Y. and Yu-Dong Y. (2003). Reverse Link Capacity of SIR-based Power Controlled CDMA Systems with Antenna Arrays. Wireless Communications and Mobile Computing. 3: 759-772.

Karim M. R. and Sarraf, M. (2002). W-CDMA and CDMA 2000 for 3G Mobile Networks, McGraw-Hill, New York.

Kim D. K. and Adachi F. (2001) Theoretical analysis of reverse link capacity of an SIR-based power-controlled cellular system in a multipath fading environment. IEEE Transactions on Vehicular Technologies. 50(2): 452-464.

Kim J. B. and Honig M. L. (2000). Resource Allocation for multiple classes of DS - CDMA traffic. IEEE Transaction on Vehicular Technologies. 49(2): 506-519.

Suyeb A. K, Jasvir S. and Mian M. (2006): Capacity Analysis of WCDMA in forward Link FDD Mode System, $3^{\text {rd }}$ IEEE Mobility Conference, organized by Rangsit University, Bangkok, Thailand, $25-27$.

Tse D. N. C. and Hanly, S. V. (1999). Linear Multiuser Receivers, Effective Interference, Effective Bandwidth and User Capacity. IEEE Transactions on Information Theory. 45(2): 641 - 657.

Xia O. M., Shroff N. B. and Chong E. K. P. (2001). Resource Management in Power - Controlled Cellular Wireless Communications and Mobile Computing. 1(2): 185-199.

Table 1. Factors which impact on WCDMA capacity and its system design parameters.

\begin{tabular}{|l|l|}
\hline Parameter & Empirical values \\
\hline Correction factor of power control $\alpha_{\mathrm{p}}$ & 2.50 \\
Voice activity factor, $v$ & 0.40 \\
Frequency reuse factor, $F_{r}$ & 0.60 \\
User data rate, $R$ & $64 \mathrm{Kbps}$ \\
$\mathrm{SNR}\left(\varepsilon_{b} / N_{0}\right)$ & $1-10 \mathrm{~dB}$ \\
Chip rate, $\mathrm{W}$ & $3.84 \mathrm{Mcps}$ \\
Capacity enhancement using MUD technique & $0.20,0.40,0.60$ \\
Adaptive antenna gain, $A_{d}$ & 1.90 \\
Loading factor, $\eta$ & 0.65 \\
Sectorization gain, $N_{s}$ & 1.50 \\
\hline
\end{tabular}




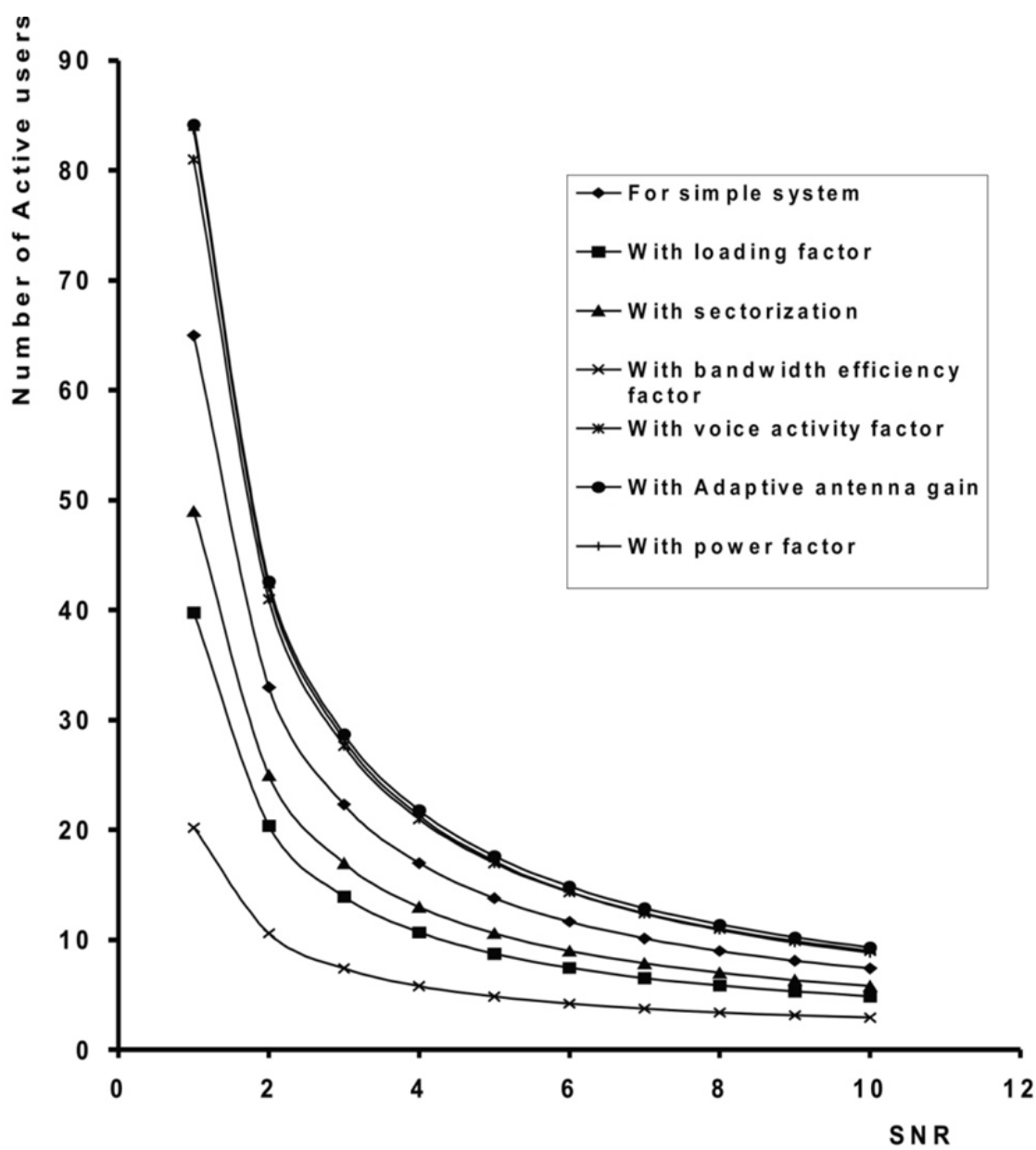

Figure 1. Effect of variable factor on users' capacity at varying SNR conditions 


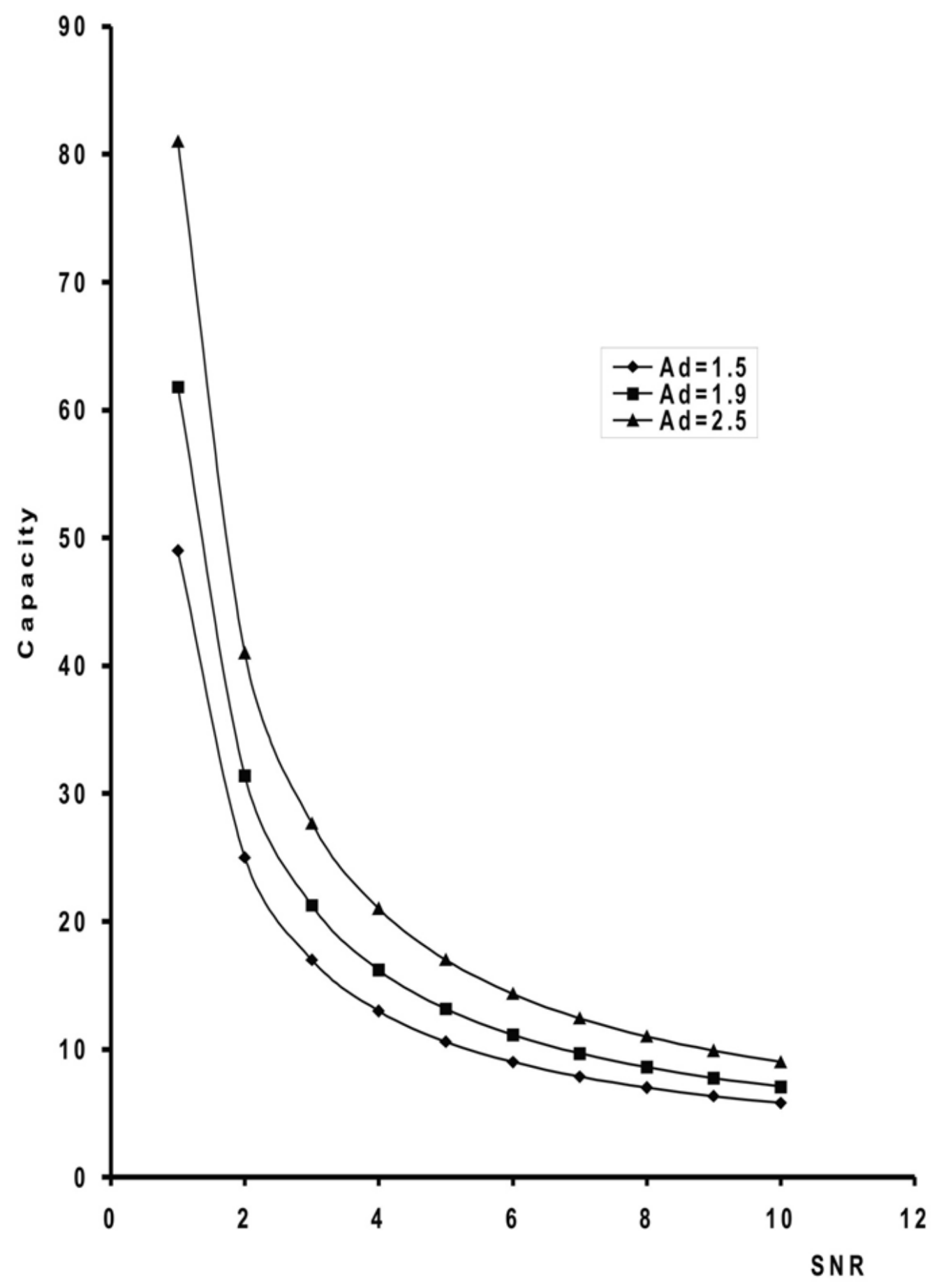

Figure 2. Comparison of different values of adaptive antenna gain (Ad) factor for voice applications on number of active users with varying SNR conditions 


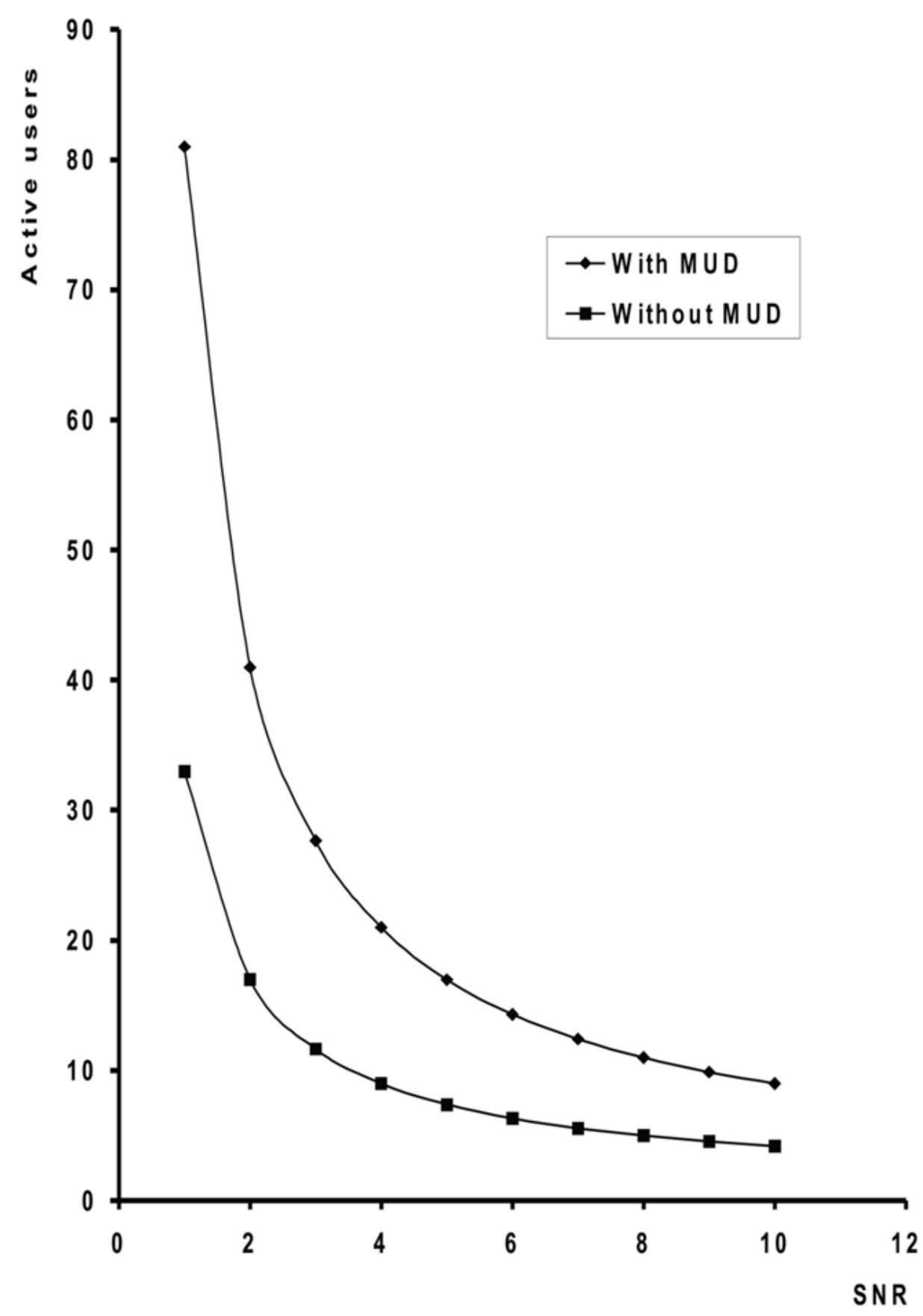

Figure 3. Effect of MUD on system capacity at varying SNR conditions for voice applications 


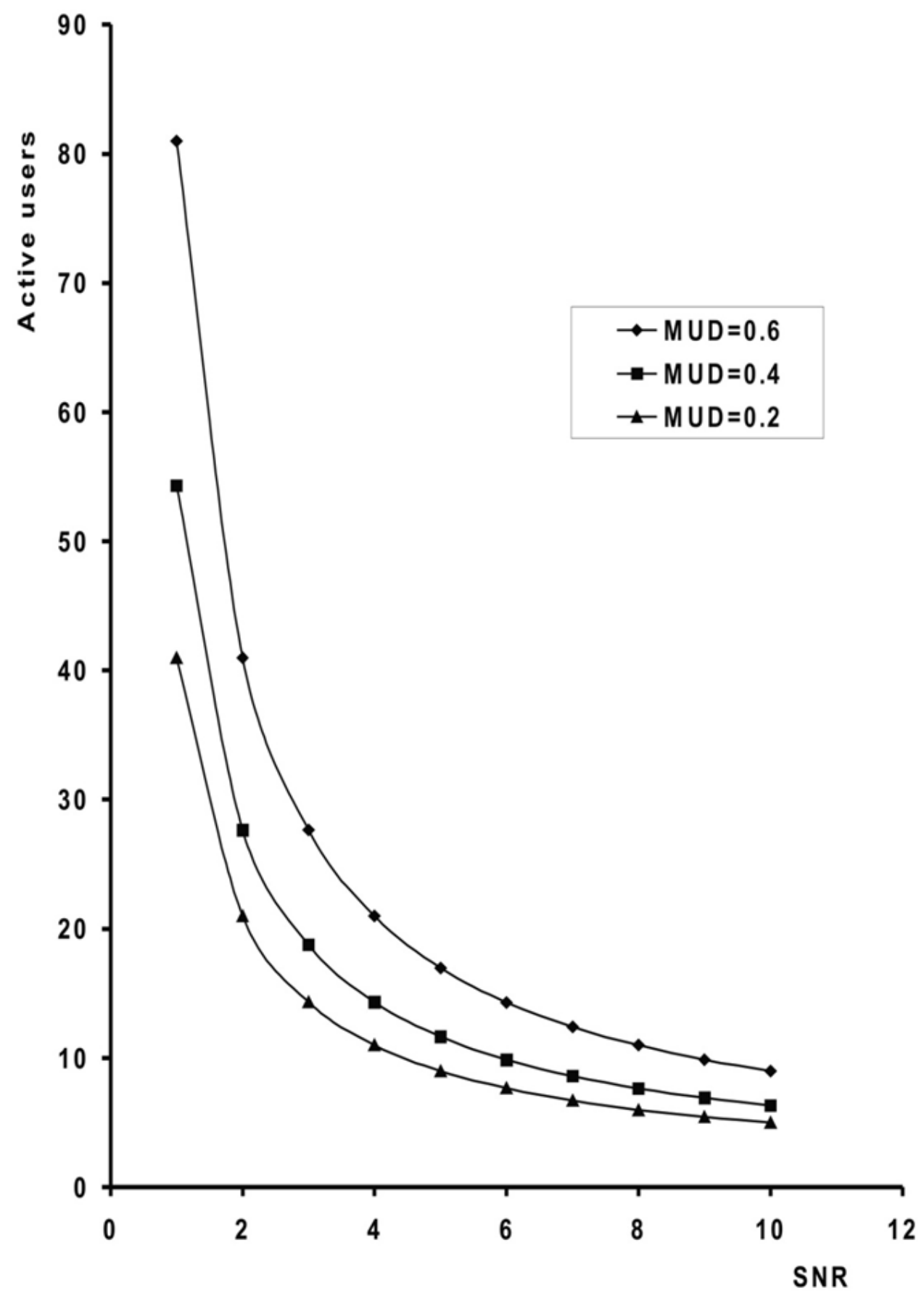

Figure 4. Comparison of different values of MUD factor with voice applications on number of active users with varying conditions of SNR 


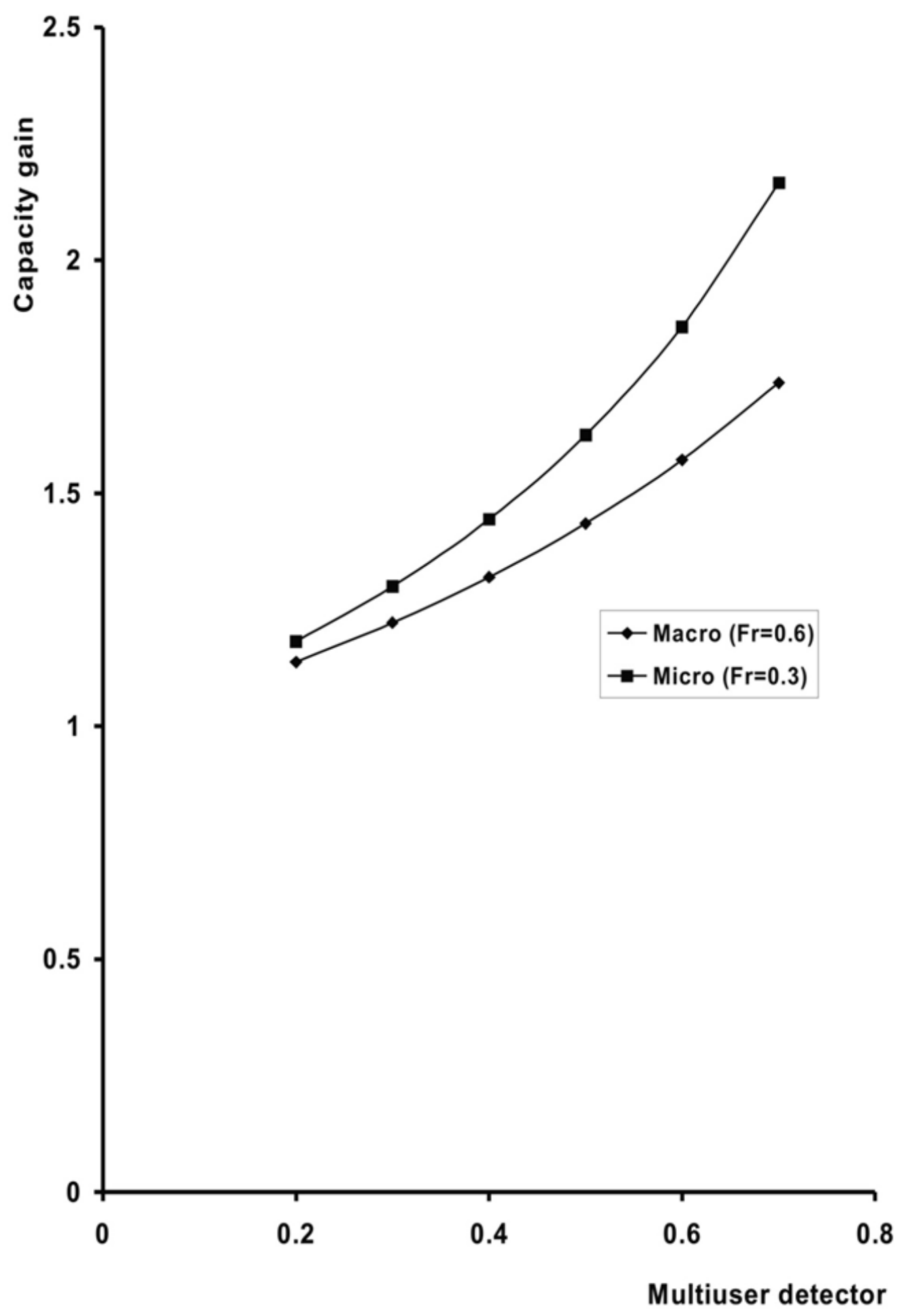

Figure 5. capacity gain with MUD for micro and macro cellular environments 


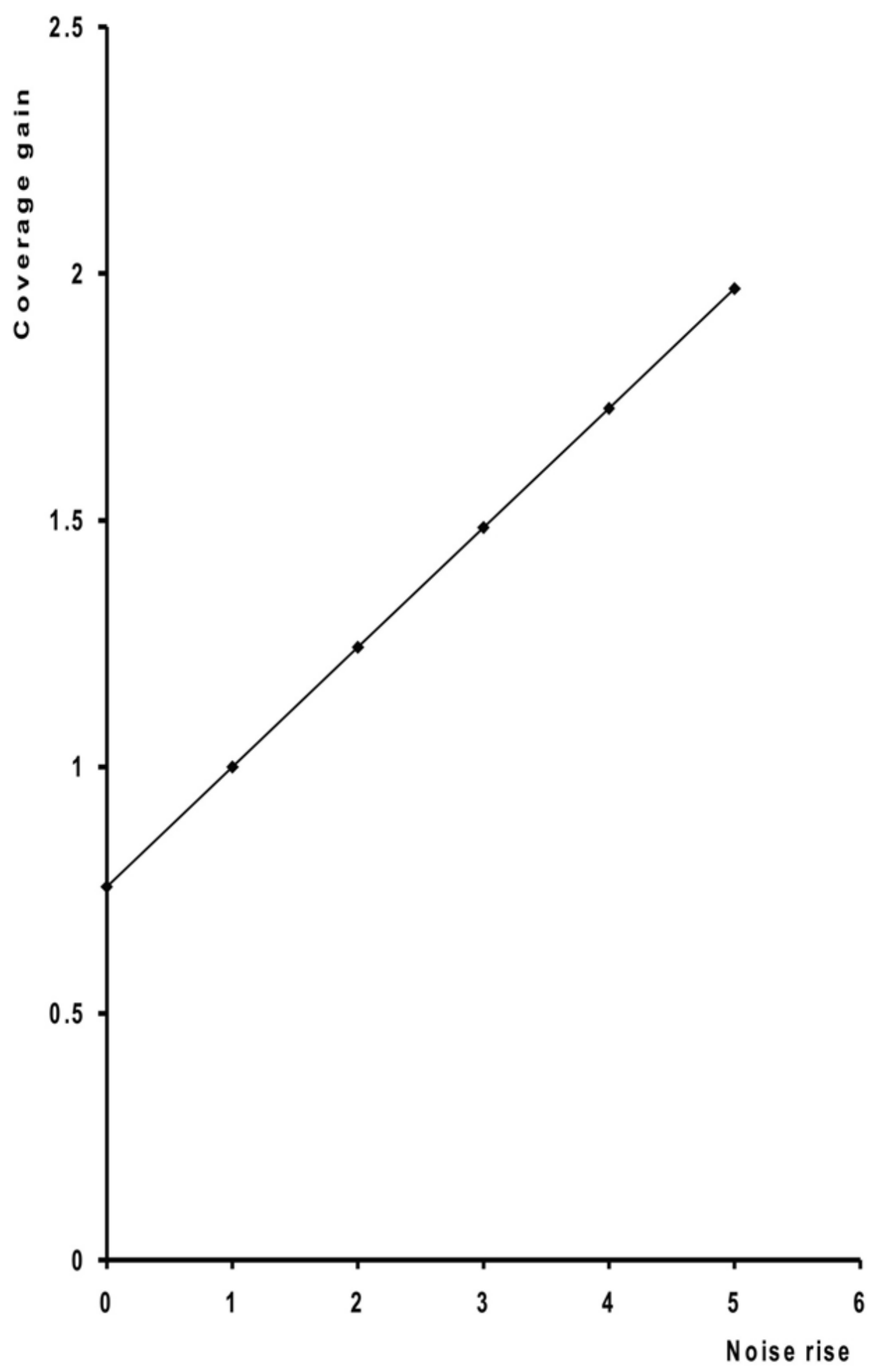

Figure 6. Coverage gain as a function of noise rise 


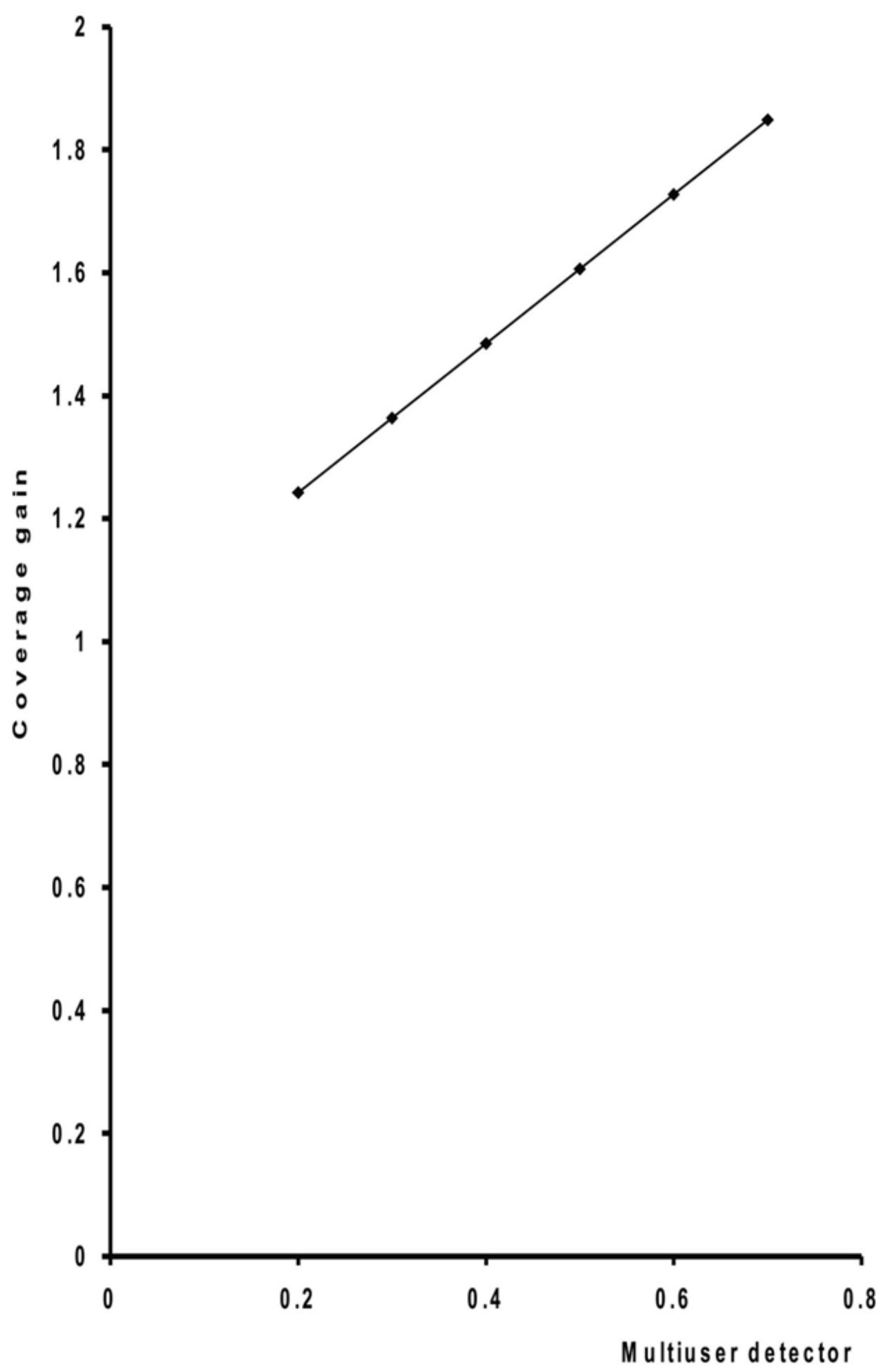

Figure 7. Coverage gain as a function of MUD 


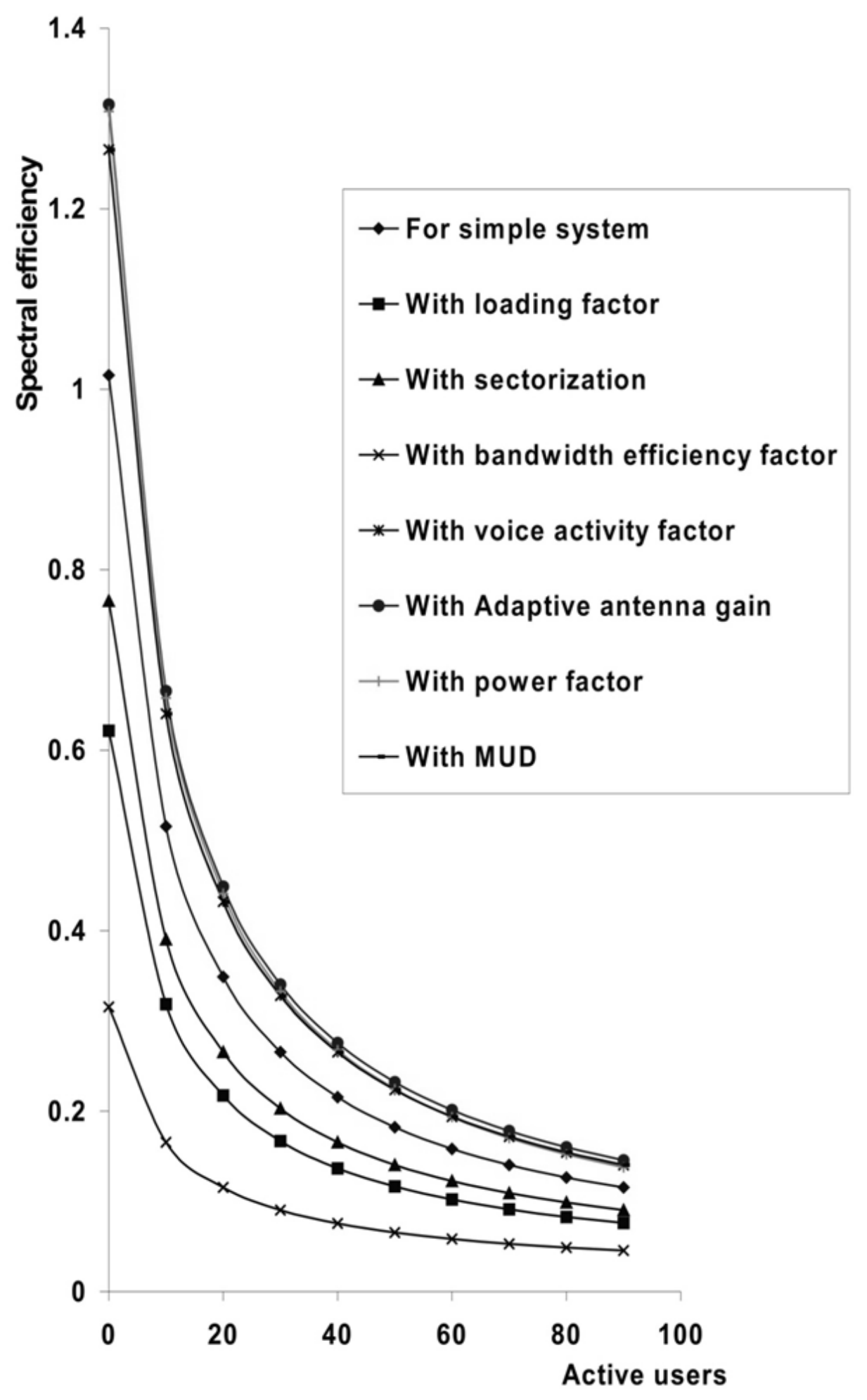

Figure 8. Spectral efficiency as a function of number of active users 